\title{
CORRIGENDUM
}

doi:10.1038/nature 17621

\section{Corrigendum: An observational radiative constraint on hydrologic cycle intensification}

\author{
Anthony M. DeAngelis, Xin Qu, Mark D. Zelinka \& Alex Hall
}

Nature 528, 249-253 (2015); doi:10.1038/nature15770

After publication of this Letter, we identified a coding error in the calculation of values that appear in Extended Data Fig. 7c and d, and which are reported in the main text. The error has the effect of overstating the broader implications of our results for a twenty-first-century climate change scenario. The calculation in Extended Data Fig. 7 represents the impact of the bias in simulated shortwave absorption on model-projected precipitation changes under the RCP8.5 scenario. The corrected values (the Supplementary Information to this Corrigendum shows the corrected Extended Data Fig. 7) indicate that removing the bias in shortwave absorption reduces the ensemble-mean precipitation increase per unit warming by $20 \%$, rather than by about $40 \%$, as stated in the first and last paragraphs of the Letter. When not normalized by warming, the reduction is $19 \%$ rather than about $25 \%$ (as stated in the last paragraph of the Letter). In the revised calculation, there is no reduction in model spread in precipitation increase per unit warming under RCP8.5 after removing the bias in shortwave absorption. This is in contrast to the $35 \%$ reduction stated in the first and last paragraphs of the Letter. However, for the precipitation increase not normalized by warming, the correction shows a spread reduction of about $10 \%$ (rather than about $25 \%$ as stated in the last paragraph of the Letter).

The lack of spread reduction in the corrected Extended Data Fig. 7c after removing the bias in shortwave absorption is mostly the consequence of two models: GISS-E2-H and GISS-E2-R (numbers 12 and 13 on the plot). These two closely related models have a large negative rapid precipitation adjustment to carbon dioxide forcing that is highly anomalous compared to other models (Extended Data Fig. 3b). These models also have the largest positive bias in temperature-mediated precipitation increase (Extended Data Fig. 7b). These two effects strongly compensate for the total precipitation increase per unit warming under RCP8.5 (see the abscissa of Extended Data Fig. 7c), such that these models do not contribute much to the spread prior to removing the bias. When the large bias in temperature-mediated response is removed, these models now have a much smaller precipitation increase per unit warming compared to other models (see the ordinate of the corrected Extended Data Fig. 7c in the Supplementary Information to this Corrigendum), contributing to substantial spread in the constrained quantity. In short, the impact of the spread in rapid precipitation adjustment, particularly from the GISS models, becomes more apparent after correcting for the shortwave absorption bias. When the GISS models are omitted from the analysis, the spread in precipitation increase, whether normalized by warming or not, decreases by about $25 \%$ after correcting for the shortwave absorption bias (see numbers in parentheses in the corrected Extended Data Fig. 7c and d). Further research is warranted to assess the cause of the large rapid precipitation adjustment in the GISS models and whether or not it is physically reasonable.

The corrected legend to the Extended Data Fig. 7 is in the Supplementary Information to this Corrigendum. The following sentences have been added: "In $\mathbf{c}$ and $\mathbf{d}$, the ratio of constrained to original model spread and ensemble mean is printed below the key. Numbers in parentheses are the ratios computed after omitting the GISS-E2-H and GISS-E2-R models (numbers 12 and 13)... 